\title{
Implicaciones de la gestión documental en México a partir de la Ley General de Archivos
}

\author{
Merizanda María del Carmen Ramírez Aceves ${ }^{1}$; Cecilia Cadena-Inostroza ${ }^{2}$
}

Recibido: 15/01/2021 / Aceptado: 03/05/2021

Resumen. En el texto se mostrará el desarrollo de la trayectoria para llegar a una Ley General de Archivos en México y las necesidades de infraestructura para su cumplimiento. La gestión documental es prioridad para implementar el Sistema Nacional de Transparencia y Anticorrupción. De los aspectos de la ley aquí se analiza la gestión documental como uno de los métodos de la Archivística fundamental para alcanzar los objetivos que plantean las políticas públicas en la materia, se aborda su interpretación, alcances, pretensiones y algunas limitaciones. A través del análisis documental y la gestión documental se llega a la conclusión de que mientras no se destinen recursos financieros y profesionales para el manejo documental, no se cumplirá la ley ni el derecho ciudadano a la información.

Palabras clave: Gestión documental, Administración de Archivos, Ley General de Archivos, Armonización Normativa Archivos y Archivo General de la Nación.

\section{[en] Implications of records management in Mexico from the General Archives Law}

\begin{abstract}
The development of the trajectory will be shown in the text to reach a General Archives Law in Mexico and the infrastructure needs for its fulfillment. Records management is a priority to implement the National Transparency and Anticorruption System. From the aspects of the law, document management is analyzed here as one of the fundamental Archival methods to achieve the objectives set out by public policies on the matter, its interpretation, scope, claims and some limitations are addressed. Through documentary analysis and document management, the conclusion is reached that as long as financial and professional resources are not allocated for document management, the law and the citizen's right to information will not be complied with.
\end{abstract}

Keywords: Record management, Archives Administration, General Archives Law, Regulatory Harmonization Archives and General Archive of the Nation.

Sumario. Introducción. 1. Introducción. 2. Contexto general. 3. La gestión documental. 3.1. Identificación documental. 3.2. Organización documental. 3.3. Valoración documental. 3.4. Descripción documental. 4. Implicaciones de gestionar los documentos en México. 5. Conclusiones. Bibliografía.

Cómo citar: Ramírez Aceves, M. M.; Cadena-Inostroza, C. (2021): Implicaciones de la gestión documental en México a partir de la Ley General de Archivos, en Cuadernos de Gobierno y Administración Pública 8-1, 15-23.

\section{Introducción}

En el entorno de la sociedad de la información el mundo necesita "ajustar sus marcos constitucionales con objeto de incorporar los nuevos derechos universales a la información". Frase que Hernández y Flores rescataban de Bovens hace unos cuantos años (Hernández y Flores, 2013: 26). Y es que, a pesar de la resistencia de muchos gobiernos por transparentar sus procesos administrativos, se comenzó desde hace un tiempo a vislumbrar el diseño y promulgación de disposiciones tendientes a legalizar los procesos de transparencia y acceso a la información en diversas latitudes. En un reporte publicado por Privacy International titulado Freedom of Information around the World 2006 se mostraba un estudio para determinar que 70 países habían publicado hasta ese momento leyes de transparencia y acceso a la información. Sorprendentemente, casi un 50\% de ellas habían entrado en vigor a partir del año 2000, (Banisar, 2006: 6) lo que reflejaba una irrupción abrupta en este contexto a inicios del siglo XXI. Hoy en día, podemos contar más de 122 países que se han sumado a este colectivo de acuerdo con datos del Global Right to Information Raiting (2019) posicionando

\footnotetext{
Universidad Autónoma del Estado de México.

mmdranireza@uaemex.mx

2 El Colegio Mexiquense, A.C.

ccadena@cmq.edu.mx
} 
la ley de México en el lugar número dos tras una evaluación de su contenido.

Las demandas sociales de acceso a la información pública, tienen como principal insumo los archivos, por lo que tales demandas han dado lugar a la promulgación de leyes en esta materia, derivado de la importancia de los documentos administrativos, en contraste con la opacidad que reinaba en épocas pasadas (y es que, desde las posturas de Izcóatl y fray Diego de Landa hasta nuestros días, la destrucción de documentos y, con ello, de información, no se han modificado). Estas disposiciones promueven el acceso a los documentos que respaldan las decisiones políticas, lo que supone una transformación profunda de la función social de los archivos. Actualmente, en los países iberoamericanos se reconoce el valor de los documentos como un medio para fomentar la identidad nacional y para establecer sociedades más informadas. Pero, la mejor ley de transparencia y acceso a la información pierde todo sentido si no existen documentos que consultar o si éstos se encuentran en tal desorden que la rendición de cuentas se vuelve imposible de ejercer. Es verdad que las leyes por sí mismas no representan una garantía para el ejercicio de este derecho, hace falta echar una mirada al pasado para hacer conciencia de las vicisitudes por las que han pasado los Archivos y observar de cerca las condiciones tan lamentables en las que se encuentran sus documentos y el lugar que ocupan en la jerarquía de la Administración Pública, por lo menos en México. En este país, a pesar de haberse creado hace cerca de dos décadas una ley de transparencia y de acceso a la información, hoy en día sigue manteniendo una precaria tradición en cuanto a gestión de documentos se refiere.

El rápido proceso de publicación de un marco legal en México que garantizara no sólo la rendición de cuentas sino la transparencia y el acceso a la información, fue consecuencia de diversos factores, entre los que se podrían mencionar la proliferación repentina de nuevos regímenes democráticos; la presión que ejercieron las instituciones financieras internacionales para que los países promulgaran este tipo de leyes; el robustecimiento que tuvieron las asociaciones regionales de libre comercio; el gran apogeo que han tenido en los últimos tiempos las tecnologías de la información y comunicación (TIC's); el impulso a las agendas anticorrupción que buscaban elevar la calidad de las Administraciones Públicas en diversas Naciones del mundo; la presión de la sociedad civil y la academia; y la implementación de estrategias de legitimación de nuevos gobiernos, entre otros.

Desde hace dos décadas en México comenzaron las discusiones en torno al derecho de acceso a la información, lo que permitió que se legislara sobre este aspecto, resultando con ello la publicación de la Ley Federal de Transparencia y Acceso a la Información Pública Gubernamental en el 2002 (H. Congreso de la Unión, 2002) y de la Ley General de Transparencia y Acceso a la Información Pública en el 2015 (Cámara de Diputados del H. Congreso de la Unión, 2015), así como de los Lineamientos para la organización y conservación de los archivos de las dependencias y entidades de la administración pública gubernamental (Archivo General de la Nación; Instituto Federal de Acceso a la Información Pública, 2004).

A partir de ello el Archivo General de la Nación elaboró una serie de herramientas que definirían una metodología para organizar, valorar y describir los documentos de archivo de este país. Pero ¿por qué siguen sin gestionarse de manera correcta los documentos de archivo? Para que los gobiernos rindan cuentas, deben promover y garantizar un ejercicio político transparente a partir de permitir el acceso a la información plasmada en los documentos de archivo que son transferidos y depositados en los archivos públicos, pues son éstos los que testimonian, evidencian e informan sobre las actuaciones de las administraciones en su día a día. A partir de la promulgación de este marco legal se comenzó a dar un fuerte impulso a los Archivos. Este contexto condujo a la generación de una Ley Federal de Archivos (H. Congreso de la Unión, 2012), que regiría la organización y conservación de los documentos que se venían produciendo desde años atrás. Recientemente quedó superada por la publicación de la Ley General de Archivos cuya regulación archivística pretende permear ya no sólo hacia el terreno federal, sino también hacia el estatal y municipal, en sus ámbitos público y privado. Esta ley fue aprobada el 26 de abril de 2018 en la Cámara de Diputados del gobierno mexicano y publicada en el Diario Oficial de la Federación el 15 de junio del mismo año, tras la iniciativa que se lanzara al Senado el 17 de noviembre de 2016. Su ejecución se vio materializada a partir del 15 de junio de 2019 con el objetivo de:

“establecer los principios y bases generales para la organización y conservación, administración y preservación homogénea de los archivos en posesión de cualquier autoridad, entidad, órgano y organismo de los poderes Legislativo, Ejecutivo y Judicial, órganos autónomos, partidos políticos, fideicomisos y fondos públicos, así como de cualquier persona física, moral o sindicato que reciba y ejerza recursos públicos o realice actos de autoridad de la federación, las entidades federativas y los municipios" (Cámara de Diputados del H. Congreso de la Unión, 2018: 1).

Aunque es una disposición legal de reciente creación e implementación que llevó muchos años de estudio para su diseño, no se ha discutido su contenido final con suficiente rigor.

Ahora bien, habría que decir que, para su estudio, la Ley General de Archivos se puede dividir en cuatro grandes ejes temáticos, que no tienen que ver necesariamente con la estructura que presenta, sino por la manera en que pueden ser estudiados los aspectos que se hallan incluidos en ella, estos son: a) gestión documental, b) administración de Archivos, c) cultura archivística y d) papel que juega el Archivo General de la Nación en este escenario. Es preciso analizar cada uno de ellos a detalle, la intención de este texto es centrar la atención en el primero, es decir, en la gestión documental, con el fin de interpretar lo dispuesto en la ley y establecer sus alcances y pretensiones. Para ello es necesario utilizar como principal método de estudio el análisis documen- 
tal clásico, empleado en la indagación exhaustiva de la literatura especializada para la creación del cuerpo teórico conceptual y metodológico de este documento. Pero al mismo tiempo, es necesario recurrir a lo que establece el propio método de la gestión documental para entender los procedimientos pragmáticos que se deben seguir para organizar de manera adecuada los documentos y, por tanto, cumplir con las políticas públicas en materia de rendición de cuentas.

El texto está compuesto de tres apartados más una introducción y unas conclusiones. En el primer apartado se aborda el contexto general, con algunos antecedentes que abrieron la posibilidad de establecer la Ley General de Archivos, así como principios generales sobre los que se sustenta. En el segundo se aborda la gestión documental como el primer paso en la producción y sistematización de información en los archivos, aquí se incluyen análisis sobre identificación, organización valoración y descripción documental.

En la tercera parte se realiza un análisis sobre las implicaciones que tiene la implementación de la ley en las condiciones actuales de los archivos en el país, resaltando algunos de los retos más visibles. Particularmente, los recursos financieros y humanos necesarios. Por último, se presenta un breve apartado de conclusiones, donde se expone el reto que tienen las administraciones públicas frente a las nuevas disposiciones normativas y las consecuencias para la eventual consolidación del Sistema Nacional de Transparencia y Anticorrupción.

\section{Contexto general}

En primer lugar, habría que precisar que la Ley General de Archivos fue publicada el 15 de junio de 2018, desplazando con ello a la antigua Ley Federal de Archivos y entró en vigor justo un año después.

Homogeneizar los procesos de organización, conservación, administración y preservación de los archivos de los sujetos obligados es la misión fundamental que plantea, así como establecer las bases para la organización y funcionamiento del Sistema Nacional de Archivos, todo ello con miras a resguardar, difundir y garantizar el acceso a la información de los ciudadanos frente a las instituciones públicas y privadas que reciban y ejerzan recursos públicos. El fin, por tanto, es administrar los Archivos y gestionar sus documentos para garantizar su acceso y difusión, cualidades que eran imposibles de materializar en el siglo XVIII con las reformas que se hicieron en el Archivo de la Secretaría del Virreinato. Sin embargo, con la implantación de los principios de igualdad y soberanía durante el siglo XIX comenzó a labrarse la idea de la difusión, lo que condujo, entre otras cosas, a crear la figura de "Archivo público". Hoy en día, la divulgación de la información es lo que más importa en los gobiernos que se precian de ser demócratas, y esta condición no es indiferente para México, al menos desde que finalizó el periodo neoliberal, pues desde la implantación constitucional del derecho de acceso a la información en 2007, hoy más que nunca se busca a toda costa la apertura de los archivos que son sus principales contenedores, tal como sucedió con los del movimiento del 68, los de la extinta Dirección Federal de Seguridad (DFS) y a la que le sucedió los del Centro de Investigación y Seguridad Nacional (CISEN), por mencionar solo algunos. Pero, para acceder a su información es necesario realizar una serie de tareas que incluyen tanto al continente (la administración del archivo), como al contenido (la gestión documental), de tal manera que se armonice la labor archivística en los tres órdenes de gobierno (federal, estatal y municipal) y en los tres poderes (Ejecutivo, Legislativo y Judicial), creando con ello una cultura archivística que pueda ser coordinada desde el órgano archivístico máximo que se materializa en la figura del Archivo General de la Nación. Y esta homologación de los procesos documentarios conduce a la implantación de un Sistema Nacional de Archivos, que implica la configuración de una metodología centrada en atender los cuatro aspectos señalados con anterioridad. Veamos cómo aborda al primero de ellos la ley en cuestión y qué implicaciones tendría para el país.

\section{La gestión documental}

La gestión documental nació como consecuencia de la progresiva adopción de los principios de calidad y de mejora continua en el ámbito empresarial y que se regularon mediante la norma ISO 9001:2000, la cual incidió directamente en el desarrollo de una norma específica sobre gestión de documentos: la ISO 15489. En este documento, se define al término como el:

control eficaz y sistemático de la creación, la recepción, el mantenimiento, el uso y la disposición de documentos de archivo, incluidos los procesos para incorporar y mantener en forma de documentos la información y prueba de las actividades y operaciones de la organización (International Standard Organization, 2001: 4).

La Ley General de Archivos tuvo preferencia por la definición que ya se había formalizado en los Lineamientos para la organización y conservación de los archivos de las dependencias y entidades de la administración pública gubernamental, que la concibe como el "tratamiento integral de la documentación a lo largo de su ciclo vital, a través de la ejecución de procesos de producción, organización, acceso, consulta, valoración documental y conservación" (Cámara de Diputados del H. Congreso de la Unión, 2018: 5) y, como se observa, no dista, en esencia, de la intención de la ISO.

La gestión documental es, por tanto, la herramienta que permite no sólo producir y sistematizar la información, sino seleccionar para su consulta ulterior la que sea de calidad y tenga precisión en sus contenidos, respetando los principios de conservación, procedencia, integridad, disponibilidad y accesibilidad, pues no se puede ejercer plenamente el derecho de acceso a la información pública si las instituciones no cuentan con una gestión documental eficaz. Este derecho constitucional implica rendir cuentas a través de la transparencia de los procesos institucionales que se manifiestan en el 
acto de documentar sus funciones. $\mathrm{Y}$ es que la actividad administrativa se distingue por su burocracia, que es obligatoria, es decir, el documentar cotidiano del actuar público que se realiza en el ámbito de las organizaciones gubernamentales, por lo que el deber de documentar constituye el sustento deontológico donde descansa el documento de archivo y rebasa el solo hecho de guardar una memoria histórica entendida esta última como "el esfuerzo de pasar de la memoria escrita por los vencedores, a la historia que articula la parte de los vencidos" (Velez, Sierra, Rodríguez y Becerra, 2016: 39).

Pero, para que esto sea efectivo, las instituciones deben contar con un marco normativo que regule formalmente sus funciones. Porque, si los miembros de las instituciones no las conocen -las funciones-, entonces ¿cómo sabrán qué documentos deben generar? En la propia ley se señala así:

Artículo 7. Los sujetos obligados deberán producir, registrar, organizar y conservar los documentos de archivo sobre todo acto que derive del ejercicio de sus facultades, competencias o funciones de acuerdo con lo establecido en las disposiciones jurídicas correspondientes (Cámara de Diputados del H. Congreso de la Unión, 2018: 7).

De allí que todo acto público ejercido por los sujetos obligados deba estar no sólo documentado y registrado, sino organizado y conservado. Pero, en primer lugar, ¿cómo documentar y registrar las funciones? Este es un cuestionamiento al que poco se ha atendido no sólo para dar una respuesta clara, sino para establecer un procedimiento metodológico común que apliquen los sujetos obligados a los que se refiere la disposición jurídica si es que la intención real es trascender hacia la homologación de los procesos archivísticos en el ámbito nacional con el fin de erradicar la dispersión de las prácticas disolutas de antaño que ponían en riesgo el patrimonio documental: "El primer gran objetivo de la regulación es la homologación de los principios y bases de tratamiento mínimo de los archivos" (INAI; AGN; UNAM, 2019: 29).

Se debe precisar que la teoría archivística ha definido agrupaciones documentales jerarquizadas de manera natural, que van desde la pieza simple, hasta el fondo documental, pasando por el expediente, la serie y la sección, que deberían generarse bajo un mismo origen funcional. Y también las hay de corte artificial en las que se circunscriben las colecciones y las secciones facticias que, desde luego, se contraponen a la naturaleza orgánica de las anteriores. De esta manera, las funciones institucionales se deben documentar siguiendo el orden que establecen estos grupos documentales. Y aquí cabría la precisión de que documentar no es registrar hechos, sino mostrar procesos, hacerlos visibles, socializarlos, involucrando una comprensión profunda de lo que la institución hizo en el pasado y hacia dónde se dirige. Documentar entonces, no es solo un ejercicio retrospectivo, sino también prospectivo. Para entender la acción retrospectiva habría que reflexionar sobre la razón de existir de los documentos. Hay que señalar que su apari- ción sobre el planeta se sitúa en el periodo protohistórico cuando las transacciones mercantiles se convirtieron en el eje económico de la sociedad que derivó en un aumento de los negocios que imposibilitaba la capacidad humana de llevar una contabilidad mental. Los documentos escritos eran entonces un buen instrumento de contabilización de las deudas por parte del acreedor en los que se asentaba la obligación de pagarlas y se verificaba su cumplimiento, en caso contrario, se procedía legalmente sobre el deudor. De allí que se consideraban la prueba fehaciente de un hecho acaecido. Y es que la acción de probar es "el procedimiento de verificación de una afirmación", es decir, aceptar un hecho. Por tanto, los documentos se convierten en un medio de prueba que cumplen funciones administrativas, jurídicas, contables y memoriales (Moreno, 2001: 10). Esto hace de ellos un elemento fundamental para el ejercicio de la rendición de cuentas y de este modo trasciende la acción prospectiva del arte de documentar.

$\mathrm{Y}$, se deben gestionar porque a lo largo del tiempo se han acumulado de tal manera que, si no se controlan con métodos archivísticos, el acceso a la información en ellos contenida se vuelve una tarea difícil. Lo que implica la aplicación de un método creado por la archivística a través del cual se puedan identificar las funciones y documentarlas con base en las agrupaciones documentales señaladas. Hay que aclarar que el método significa etimológicamente "ir a lo largo del camino" (Bochenski, 1981: 28), esto es, un trayecto que se recorre. Aplicar un método implica entonces actuar de manera racional y con un orden trazado previamente para alcanzar un objetivo determinado. Es así como el análisis detallado de cada una de las funciones institucionales llevaría a recorrer un camino rutinario, secuencial y ordenado que va desde saber dónde inicia un procedimiento y dónde termina para determinar cada una de las funciones señaladas e ir derivando de cada parte de ese procedimiento los documentos que se deben generar. Por esta condición, las categorías funcionales de la institución se interrelacionan y se cohesionan con las categorías archivísticas, aplicando así un método al que se podría denominar método de identificación documental.

\subsection{Identificación documental}

La identificación fue definida en los años noventa y reconocida en el Diccionario de Terminología Archivística como:

el proceso de investigación y sistematización de categorías administrativas y archivísticas en las que se sustenta la estructura de un fondo, siendo uno de sus objetivos principales asegurar a través de sus resultados la valoración de las series documentales (Sudirección General de los Archivos Estatales, 1995, s/p).

Este proceso implica identificar las funciones de cada una de las áreas de la institución, pues ellas se verán materializadas en los documentos y provocarán su producción. Por lo tanto, hay una correlación directa e indisociable entre el marco legal institucional-que es el que 
formaliza las funciones- y las agrupaciones documentales naturales principalmente. Así pues, en la medida en que las funciones estén redactadas de forma clara, no haya omisiones de ninguna de ellas, no se dupliquen o no se hallen contenidas en más de una, en esa medida se generarán fondos documentales bien estructurados. Con ello se garantizará que a los documentos se les dote de los elementos de identificación necesarios para asegurar que mantengan su procedencia y orden original (Cámara de Diputados del H. Congreso de la Unión, 2018, Ley General de Archivos, artículo 11, inciso VI), premisas fundamentales que desde el siglo XIX ya venían proponiéndose y que a lo largo del tiempo han generado diversos estudios que concluyen al unísono en la postulación de éstos como principios bajo los cuales se debe regir la labor archivística. Los holandeses Samuel Muller, Johan A. Feith y Robert Fruin los defendieron en las reglas 16 y 17 de su Manual de la siguiente manera:

16. El sistema de arreglo debe basarse en la organización original de la colección de archivo, que en general corresponde a la organización del cuerpo administrativo que lo produjo

17. En la organización de una colección de archivos, por lo tanto, el orden original debe, en primer lugar, restablecerse en la medida de lo posible. Solo después de eso puede uno juzgar si, y hasta qué punto, es deseable desviarse de ese orden (Muller, Feith, \& Fruin, 2003: 52-59).

Por lo tanto, la identificación - primera tarea de la gestión documental- representa la manera de "documentar" la información institucional.

Por otro lado, documento no es sinónimo de registro. La norma ISO 15489 define a éste último como "el acto por el que se atribuye a un documento de archivo un identificador único al introducirlo en un sistema" (International Standard Organization, 2001: 4) El...

"registro consiste en dar testimonio de la creación o incorporación de un documento en un sistema de gestión de documentos de archivo; lo cual, como ventaja adicional, facilita su recuperación. Consiste en la introducción de una breve información descriptiva o de metadatos sobre el documento de archivo y la asignación de un identificador único dentro del sistema. El registro formaliza la incorporación del documento al sistema" (International Standard Organization, 2001: 20).

El registro, que puede ser de documentos entrantes o salientes de una entidad administrativa, supone "la asignación de un número, código o identificador único dentro del sistema, que normalmente va acompañado de una breve información descriptiva que identifica el documento" (Bustelo, 191?: 23). Permite, por tanto, dejar constancia de que un documento de archivo fue creado, formalizando con ello su incorporación al fondo documental. Por lo tanto, primero se documenta una función teniendo como resultado un documento de archivo y después se deja evidencia de su creación en un registro.
Como se señaló anteriormente, todo acto público, además de ser documentado y registrado, debe también ser organizado y conservado. Pero la organización documental y la conservación no representan tareas menores ni se traducen en la minimizada visión estereotipada de almacenamiento de papeles. En primera instancia, la organización supone una serie de procesos tendientes a clasificar y ordenar un fondo documental. Este término se ha estudiado con tanto rigor que ha derivado en asumir posturas diferenciadas entre quienes aceptan solo dos tareas como parte de la organización "La organización archivística es el resultado de las operaciones de clasificación e instalación de los documentos". Y hay quienes en ésta misma consideran a la descripción documental "La organización de los documentos de archivo es el resultado de las operaciones de clasificación, descripción e instalación" (Barbadillo, 2007: 33). A decir verdad, parecería que las variaciones en la enumeración de los procesos son intrascendentes, pero en realidad, y tras muchos años de estudio, se refuerza la idea de que la gestión documental demanda una secuencia lógica y sistemática en el arreglo de los documentos que implica la identificación, la organización, la valoración y la descripción. Y durante este trayecto se estarían observando los mecanismos de conservación.

\subsection{Organización documental}

La organización es definida como "la operación por la que se agrupan los documentos individuales (piezas documentales) en unidades inteligibles (series) y estas unidades entre sí a fin de quedar relacionadas" (Gallego, 1989: 82). Lo que supone una estructura sistematizada y escalonada de interrelación de documentos en concordancia con las agrupaciones documentales definidas con antelación.

Esta tarea reúne tanto una operación intelectual (clasificación), como una mecánica (ordenación o instalación).

La clasificación es un método de conocimiento e información que consiste en crear grupos -documentos en este caso- lo más homogéneos posible y supone divisiones jerárquicas o no, materializándose en el Cuadro General de Clasificación Archivística (instrumento de obligatoriedad según la Ley) que da pauta a ordenar o instalar físicamente los documentos en estantería. Así, la ordenación supone relacionar unos documentos con otros de acuerdo con un método previamente establecido que puede ser por fecha (cronológico), letras (alfabético), números (numérico) o asuntos (por concepto). De allí entonces que la organización sea una operación intelectual y mecánica por la que las diferentes agrupaciones documentales se relacionan de forma jerárquica con criterios orgánicos y funcionales para revelar su contenido e información.

\subsection{Valoración documental}

A esto le debería seguir uno de los pasos más trascendentales y, al mismo tiempo, más sensibles, de la metodología archivística: la valoración documental, porque a 
partir de ésta se determina lo que en el futuro será estudiado acerca del pasado, recayendo en ello una vez más la acción retrospectiva y prospectiva de la información documentada.

La valoración como función y proceso archivístico, tiene como propósitos centrales identificar y asignar los valores primarios (administrativos, legales y fiscales de la documentación) y sus eventuales valores secundarios (evidenciales, testimoniales e informativos), a fin de establecer con base en estos, los tiempos de vigencia o resguardo de la documentación en la estructura de archivos de un Sistema Institucional, (INAI; AGN; UNAM, 2019: 56)

Consiste pues, en el establecimiento de valores documentales, vigencias, plazos de conservación y disposición de las series documentales, que se objetivan en un Catálogo de Disposición Documental, instrumento que también deben elaborar los sujetos obligados por la ley, debiendo considerar para su confección los principios de procedencia y orden original, así como el criterio diplomático, de contexto, de contenido y de utilización.

Hay que recordar que la valoración no siempre ocupó un lugar de reconocimiento en el marco disciplinario, antes bien, obtuvo su legitimación en el momento posterior a la Segunda Guerra Mundial. Quienes defendieron por primera vez esta acción fueron Alemania e Inglaterra. El primer país abanderó la idea de "valorar para conservar" y descansó su labor en dos referentes fundamentales, por un lado, la importancia del organismo productor y, por otro, la usabilidad de los documentos. Los ingleses, en cambio, trabajaban bajo la premisa de "valorar para eliminar", por lo que esta tarea se ejercía bajo los intereses meramente administrativos. Cuando la valoración llegó a América, Estados Unidos asumió la idea jenkinsoniana de mantener a los archivistas al margen de esta práctica, sin embargo, el cada vez menos ralentizado crecimiento de las masas documentales orilló a descansar en ellos esta labor que, en un principio, era exclusiva de los propios productores de documentos. De esta manera, la valoración comenzó a tomar una posición estratégica no solo en el vecino del norte, sino que pronto se extendió al resto del continente americano. Así, la atribución de valores a los documentos comenzó a solidificarse. La importancia de los documentos para la institución productora, para el estudio de la historia administrativa y para la historia en general fueron las cualidades que propuso Philips Brooks aunado al establecimiento de valores primarios y secundarios de Theodore Shellenberg (Del Castillo y Ravelo, 2017: 276). Estos elementos permitieron instrumentar una metodología en torno a la valoración que ha condicionado la propia praxis archivística a tal grado que ha originado el establecimiento de diversos modelos en diferentes latitudes territoriales.

En la historia mexicana se destacan diversos acontecimientos que muestran una frecuente inconsistencia en materia de Archivos y que hoy concluyen en la creación de grupos interdisciplinarios en cada sujeto productor que serán los responsables de determinar el valor de los documentos de acuerdo con los principios y criterios señalados con anterioridad.

Ahora bien, los entornos digitales actuales introyectaron en la Archivística una nueva forma de entender no sólo la realidad social, sino las actuaciones institucionales y proponer nuevos atributos de valoración documental encaminados a atender la validez, autenticidad, inmediatez, seguridad, trazabilidad y accesibilidad. Entre ellos destacan los principios generales propuestos en los años noventa por Charles Dollar y más tarde por David Bearman centrados en analizar las propias funciones institucionales; en el deber de los archivistas por establecer los requisitos esenciales para la gestión documental; el de incluir la valoración en la fase de diseño de los sistemas informáticos y; la ejecución automática de los metadatos en las diferentes etapas del ciclo vital (Dollar, 1992). A partir de allí las propuestas no han cesado.

Así pues, desde el surgimiento de esto que se enmarca en la época del paradigma postcustodial -que dio inicio en la década de los ochenta-, la noción del valor del documento se ha centrado fundamentalmente en la representación misma de los valores emanados de la propia sociedad, así lo manifestaba Booms (1987: 106) al señalar que "si hay de hecho cualquier calificado para prestar legitimidad a la valoración archivística es la propia sociedad, y las opiniones públicas que expresa. El público como un elemento constitutivo de la sociedad moderna (...) genera el proceso socio-político y legitima la autoridad política" (Booms, 1987: 106). De esta manera, el contexto social y sus valores plurales son el marco de referencia en el que se circunscriben los criterios actuales de valoración de los documentos tanto físicos como electrónicos.

\subsection{Descripción documental}

La gestión documental finaliza con el proceso descriptivo y hay que recordar que éste también parte de la aplicación de un método. El método descriptivo es la exposición narrativa, numérica, gráfica o de cualquier otra índole que se hace lo más detallada y exhaustiva posible sobre la realidad que se investiga, en este caso, de la información contenida en los documentos de archivo. A partir de la descripción se elaboran los instrumentos que facilitan el conocimiento y la consulta de los documentos para diversos fines. Describir un documento significa enumerar sus cualidades y los elementos fundamentales para su reconocimiento. Antonia Heredia la definió como "el análisis que hace el archivero sobre los fondos y los documentos de archivo agrupados de forma natural o artificial, a fin de sintetizar y condensar la información contenida en los mismos" (Heredia, 1995: 143). La descripción se aplica con la finalidad de crear representaciones de las agrupaciones documentales, es decir, sustitutos en que se registra la información archivística sobre sus atributos y sus relaciones. Este procedimiento ha llevado consigo un esfuerzo colaborativo de normalización a nivel internacional, originando normas como la ISAD (G) y las ISAAR (CPF) que regulan la descripción de los documentos de archivo y la de instituciones, personas y familias respectivamente. Esta regulación 
deberá expresarse en los instrumentos de control (cuadro general de clasificación archivística y catálogo de disposición documental) y consulta (inventario general, de transferencia y de baja documental) establecidos en los comentarios de la Ley General de Archivos (INAI; AGN; UNAM, 2019: 79).

En este mismo documento se señala que la elaboración de estos instrumentos favorecerá las subsecuentes actividades archivísticas tendientes a favorecer el acceso a la información y ejercer el "delicado proceso de valoración documental” (INAI; AGN; UNAM, 2019: 53).

Estos son los elementos correlativos que se proponen para el cometido que plantea la gestión documental, la cual se convierte en una metodología, en un conjunto de pasos consecutivos que, de aplicarse siempre de la misma manera, tendrán el mismo resultado. La misma ley la define como "el tratamiento integral de la documentación a lo largo de su ciclo vital, a través de la ejecución de procesos de producción, organización, acceso, consulta, valoración documental y conservación" (Cámara de Diputados del H. Congreso de la Unión, 2018, General de Archivos, artículo $4^{\circ}$, inciso XXXIV). La doctrina archivística establece cuatro tareas para esta metodología: identificar, organizar, valorar y describir, (Ramírez, 2014: 35) para entonces acceder y consultar. Y este uso permanente de los documentos va mermando su integridad física, por lo que hay que implementar mecanismos de conservación. Este elemento es definido en la propia ley como el "conjunto de procedimientos y medidas destinados a asegurar la prevención de alteraciones físicas de los documentos en papel y la preservación de los documentos digitales a largo plazo" (INAI; AGN; UNAM, 2019: 36).

La conservación implica considerar tanto la edificación de los espacios geográficos para el almacenamiento de los documentos físicos, como el mantenimiento de los ciberespacios para los documentos electrónicos en donde la preservación digital, la vigilancia tecnológica y la ciberseguridad de la información juegan un papel trascendental.

\section{Implicaciones de gestionar los documentos en México}

¿México cuenta con la infraestructura necesaria para gestionar sus documentos? Si se parte de la idea de que son los archivistas profesionales los capacitados para la implantación correcta del método entonces el panorama se presenta desolador si se muestran las cifras recientes y prometedor si se proponen estrategias de solución. Con relación a la parte desagradable se acude a las cifras presentadas en el 2014 por el Instituto Nacional de Estadística, Geografía e Informática (INEGI) en donde establecía, para ese año, la existencia de más de 2,643 instituciones que generan de manera directa o indirecta bienes públicos, es decir, una oferta de satisfactores que se traducen en tangibles o de servicios. En ese documento solo se señalan aquellas que fueron posibles de identificar bajo una perspectiva jurídico-administrativa (171 del Poder Judicial y Legislativo, 200 del gobierno o dependencias federales, 194 empresas públicas y paraestatales, 313 escuelas, hospitales, clínicas y servicios asistenciales, 1,122 de los gobiernos estatales y 53 de gobiernos municipales) y las ubicadas en un plano económico-funcional, esto es, las que reciben financiamiento del gobierno (228 de banca de desarrollo, fondos, fideicomisos y patronatos, 40 instituciones autónomas de educación, 31 organismos autónomos, 54 iglesias, asociaciones profesionales, cámaras o sindicatos, 150 asociaciones civiles, 43 organismos internacionales, 44 partidos políticos, (INEGI, 2014: 1-123). Esas 2,643 instituciones (y sus diversas y complejas estructuras) se traducen en la existencia de la misma cantidad de archivos en el sector público.

Un indicador contra el que se deben confrontar estas cifras es la existencia de tan solo siete instituciones de educación superior que forman profesionales en gestión documental $^{3}$. Si bien la mayoría de esas instituciones ofrecen carreras de biblioteconomía, en general están acompañadas de gestión de documentos y archivos, a excepción de la Universidad Nacional Autónoma de México, la Escuela Nacional de Biblioteconomía y Archivonomía del Instituto Politécnico Nacional y la Universidad Autónoma de San Luis Potosí que en los títulos de sus licenciaturas incluyen explícitamente la archivística. Por lo tanto, existe una clara carencia de profesionales para las nuevas y complejas tareas de los archivos, si consideramos la cantidad de archivos en todo el país y órdenes de gobierno que según la ley se deben gestionar. Peor aún si, además, tomamos en cuenta la estructura organizacional a la que se aspira desde la propia Ley General de Archivos y que consiste en que en cada organización deberá crearse un Sistema Institucional de Archivos compuesto por un coordinador general, uno para el área de correspondencia, uno por cada archivo de trámite existente, uno para el de concentración y uno más para el histórico, (Cámara de Diputados del H. Congreso de la Unión, 2018, Ley General de Archivos, artículo 21, inciso II) lo que significa que la cantidad de archivistas especializados requeridos debería ser por lo menos cinco veces más de los que existen en la acutalidad.

Esta es una de las numerosas razones que explica que, al vencimiento de la entrada en vigor de la Ley de Archivos, que se completó el 15 de junio de 2019, no se hubiera avanzado en las tareas de homologación y armonización de las leyes de los estados, que deberían estar listas en junio de 2020. Según la ley, en esta fecha las entidades federativas debían demostrar que sus leyes garantizan la regulación de sus sistemas y consejos locales, la regulación, el funcionamiento y las atribuciones de su ente estatal especializado en archivos, así como los principios de conservación, procedencia, integridad, disponibilidad y accesibilidad. Puesto que se trata de una ley general, como ya se mencionó, hay cambios para

Se trata de la Universidad Nacional Autónoma de México, la Universidad de Guadalajara, la Universidad de Nuevo León, Universidad de Chiapas, Universidad Autónoma de San Luis Potosí Universidad Autónoma del Estado de México y la Escuela Nacional de Biblioteconomía y Archivonomía del Instituto Politécnico Nacional, consultado en https://universidadesdemexico.mx/universidades/institutopolitecnico-nacional/licenciatura-en-archivonomia, recuperado el 28 de julio de 2020 . 
toda la administración pública federal, estatal y municipal, al igual que sus tres poderes, ejecutivos, legislativo y judicial.

Según información disponible en la página del Archivo General de la Nación, ente que centraliza y debe articular las tareas de la organización de archivos, al 1 de julio de 2020, es decir más de un año después del plazo para que las entidades del país armonizaran sus leyes con la Ley General de Archivos, solo ocho de ellas lo han realizado. Cuatro estados más la han aprobado, pero no han publicado su ley Estatal de Archivos ${ }^{4}$. El paso siguiente a la armonización de las leyes estatales es la creación de un Consejo Estatal de archivos, mismo que debía estar formado seis meses después de la armonización, en la actualidad solo cuatro entidades lo han hecho. Es decir, existe un rezago de más de un año y medio en la implementación de acciones que manda la Ley. El Archivo General de la Nación solo menciona que ha hecho llamados a las entidades para que cumplan con la normativa.

Todo lo anterior implica además de un esfuerzo político para emprender la armonización de las leyes, una tarea de actualización y organización de archivos, pero también de, como se ha dicho antes, evaluación e integración del proceso documental. Es un trabajo mayúsculo de acuerdo a la amplitud de organizaciones públicas y de órdenes de gobierno que comprende la ley, aunado a que la mayoría de ellos presentan disparidad en la presentación de sus archivos.

Pero quizá uno de los retos más importantes es otorgar a la tarea documental su carácter profesional, cultural y técnico. Pues si los archivos se siguen considerando, como hasta la actualidad, espacios de castigo para los administradores públicos la ley no se cumplirá y ni la memoria, ni la documentación del quehacer cotidiano y con ello de los derechos de los ciudadanos para acceder a la información podrá hacerse realidad. Porque el insumo fundamental del acceso a la información seguirá ausente.

\section{Conclusiones}

México es un país que a pesar de contar con un rico patrimonio documental heredado de la época colonial

\footnotetext{
Los estados que armonizaron sus leyes son: Zacatecas, Colima, Durango, Nuevo León, Hidalgo, Jalisco, Oaxaca y Yucatán; los que la aprobaron pero no la han publicado: Ciudad de México, Tlaxcala, San Luis Potosí y Guanajuato. Las entidades que ya cuentan con su Consejo Estatal de Archivos son: Durango, Zacatecas, Jalisco y Oaxaca, véase https://www.gob.mx/agn/articulos/agnmex-brindaacompanamiento-a-entidades-federativas-para-la-armonizacion-eimplementacion-de-la-ley-general-de-archivos, recuperado el 24 de agosto 2020 .
}

no ha logrado crear una infraestructura adecuada para evitar que se siga destruyendo debido a sus malas condiciones de conservación en la mayoría de los casos. Sin embargo, se han hecho esfuerzos considerables, y uno de ellos ha sido la creación reciente de un marco legal que, de manera paulatina, ha permeado en todas las entidades federativas. Sin embargo, las leyes por sí mismas no representan un cambio si no existe un andamiaje estructural y de capital humano que garantice su aplicación y cumplimiento.

El factor económico no es menos importante, en 2018 se aprobó un presupuesto de alrededor de $85 \mathrm{mi}$ llones de pesos, poco menos de 18 millones para la operación del Archivo General de la Nación y 67 millones para el pago de sueldos, remuneraciones y prestaciones para los trabajadores. Una dependencia que, antes de la ley atendía documentos de 300 sujetos obligados y con la nueva disposición debe estar pendiente de más de 12 mil (Diario Oficial de la Federación, 19 de junio 2020) no puede operar los cambios y cubrir las nuevas necesidades con ese presupuesto. Por lo que, para cumplir con las exigencias que demanda la ley, en 2019 debía contar con un presupuesto de $470 \%$ más que el aprobado el año anterior (Camacho, 2018). Este porcentaje da cuenta a pinceladas del presupuesto que demanda el mantenimiento, organización y puesta a disposición de la ciudadanía de los documentos que forman parte de la memoria histórica de este país y son la base para el ejercicio de un derecho democrático como lo es el acceso a la información y la consecuente demanda de rendición de cuentas.

Por otro lado, es paradójico que el Archivo General de la Nación, siendo una de las instituciones más longevas de México, creada en 1823 durante el Virreinato, haya esperado cerca de 200 años para promulgar una ley en materia de archivos. Más sorprendente resulta que, existiendo toda una teoría que ha marcado las bases de la gestión documental a nivel internacional, no se haya aplicado en los archivos de este país.

Finalmente, hay que decir que es necesario, además de la obligación de los estados de armonizar la ley y así garantizar un derecho a los habitantes, la contratación de personal especializado es un elemento crucial, pues por el manejo y tratamiento de documentos es evidente que se trata de un trabajo profesionalizado y que no cualquier funcionario puede realizarlo. Se necesita claridad y experiencia en la gestión documental, de otro modo no logrará construirse el pilar que todavía falta para el establecimiento del Sistema Nacional de Transparencia y con ello las condiciones para el ejercicio del derecho ciudadano a saber. 


\section{Bibliografía}

Archivo General de la Nación; Instituto Federal de Acceso a la Información Pública. (2015). Lineamientos generales para la organización y conservación de los archivos de las dependencias y entidades de la administración pública federal. México: Diario Oficial de la Federación.

Banisar, D. (2006). Freedom of information around the world 2006: A global survey of access to government information laws. Estados Unidos: Privacy International. Disponible en: https://ssrn.com/abstract=1707336 or http://dx.doi.org/10.2139/ ssrn.1707336. (Consultado el 23 de agosto de 2020).

Barbadillo Alonso, J. (2007). “Apuntes de clasificación archivística”, Legajos. Cuadernos de investigación archivística y gestión documental, 10: 27-50.

Bochenski, J. Mª (1981). Los métodos actuales del pensamiento. España: RIALP.

Booms, H.; Joldersma, H y Klumpenhouwer, R. (1987). "Society and the formation of a documentary heritage: Issues in the appraisal of archival sources", Archivaria 24: 69-107.

Bustello, C. (201?). Procesos de creación y control de documentos. Cataluña: Universitat Oberta de Catalunya. Disponible en: https:/www.exabyteinformatica.com/uoc/Informatica/Procesos_y_herramientas_de_gestion_documental/Procesos_y_ herramientas_de_gestion_documental_(Modulo_1).pdf (Consultado el 27 de abril de 2021).

Camacho, Zósimo. (2018). “Ärchivo General de la Nación, rebasado por sus nuevas responsabilidades”, Contralinea, Disponible en: http://www.contralinea.com.mx (Consultado el 13 de febrero de 2020).

Cámara de Diputados del H. Congreso de la Unión. (2002). Ley Federal de Transparencia y Acceso a la Información Pública Gubernamental. Ciudad de México: Diario Oficial de la Federación.

Cámara de Diputados del H. Congreso de la Unión. (2015). Ley General de Transparencia y Acceso a la Información Pública. Ciudad de México: Diario Oficial de la Federación.

Cámara de Diputados del H. Congreso de la Unión. (2018). Ley General de Archivos. Ciudad de México: Diario Oficial de la Federación.

Del Castillo, J y Ravello Díaz, G. (2017). "El proceso de valoración documental a la luz de los actuales debates archivísticos", Revista Interamericana de Bibliotecología, 40 (3): 273-283.

Dollar, C. (1992). Archival theory and information technologies. The impact of information technologies on archival principles and methods. Macerata: University of Macerata.

Gallego Domínguez, O. (1989). Introducción a la archivística. Vitoria: Servicio Central de Publicaciones del Gobierno Vasco.

Heredia Herrera, A. (1995). Archivística general. Teoría y práctica. Sevilla: Diputación Provincial de Sevilla.

Hernández Quiñones, A y Flórez Herera, J. (2013). Accountability social y democracia: el caso de la Red Latinoamericana por Ciudades Justas, Democráticas y Sustentables. Bogotá: Universidad de los Andes.

Instituto Nacional de Estadística, Geografía e Informática. (2014). Catálogo de Dependencias e Instituciones de Interés Público. Ciudad de México: INEGI.

International Standard Organization. (2001). Norma Internacional ISO 15489-1. Ginebra, Suiza: ISO.

Moreno Navarrete, M. A. (2001). La prueba documental. Estudio histórico-jurídico y dogmático. Madrid: Marcial Pons.

Muller, S., Feith, J., Fruin, R. (2003). Manual for the arrangement and description of Archives. Illinois: Society of American Archivists.

Ramírez Aceves, M. (2014). Gestionar los documentos de archivo para garantizar el acceso a la información. México: UAEMéx. Sudirección General de los Archivos Estatales. (1995). Diccionario de terminología archivística. Madrid: Ministerio de Cultura.

Velez, O. C., Rodríguez Oar, A., Sierra, A. Ma., y Becerra, S. (2016). "La memoria histórica en los procesos de acompañamiento pastoral a personas en situación de desplazamiento", Veritas. Revista de Filosofía y Teología, 34: 33-60. 
